\title{
Kepler, Snowflakes, and Pomegranates
}

In late December of 1610, the German astronomer and mathematician Johannes Kepler walked the streets of Prague, returning home after a visit to his "master and benefactor," the "illustrious Counsellor at the Court of His Sacred Imperial Majesty," Johannes Matthäus Wacker von Wackerfels. As Kepler crossed the Karlsbrucke Bridge, he noted his embarrassment at not having a New Year's gift for his benefactor.

"Just then by a happy chance watervapor was condensed by the cold into snow, and specks of down fell here and there on my coat, all with six corners and feathered radii," Kepler wrote later in a short treatise. "Pon my word, here was something smaller than any drop, yet with a pattern; here was the ideal New Year's gift... since it comes down from heaven and looks like a star."

The experience hardly seems like one of scientific significance. However, Kepler turned it into one. Reaching home, he wrote down his ruminations on the hexagonal shape of snowflakes in an essay titled A New Year's Gift, or On the Six-Cornered Snowflake, which he presented to Wacker shortly thereafter. Full of wit and contemplation of the mystical "formative faculty" (a property of the Earth embodying a universal spirit that shapes everything) that Kepler employed to account for the genesis of forms, it also contained a complete discussion of the orderly arrangement of spheres when packed together. As the mathematical physicist and philosopher of science Lancelot Law Whyte wrote in the introduction to a 1966 English translation of the work, "Kepler's essay provides the first published evidence, in diagrams as well as text, of the ideas of regular arrangements and close-packing which have proved fundamental to crystallography." Whyte noted that the Englishman Thomas Harriot wrote a manuscript in 1600 dealing with the same issues, but it was never published.

How did a winter's walk and a few stray snowflakes lead to such valuable scientific deductions? Kepler allowed his mind to wander along with his legs, leading him to consider honeycombs and pomegranates in addition to snowflakes. In the end, it was his investigation of the pomegranate, with its inner chambers, or "loculi," as Kepler called them, which yielded the most important results from a materials science perspective.

Initially, he assigned the cause of the shape of the loculi to the plant's soul, or "life principle." However, he noted that the life principle "is not a sufficient cause of its shape: for it is not from its formal properties that it induces this shape in its fruit, but it is assisted by material necessity [italics added]." The loculi are very small and round to begin with, he said, encased in a soft rind. But as the loculi grow and the rind hardens, they become squeezed into the available space. The most efficient packing of spheres is thus a major consideration.

"How did a winter's walk and a
few stray snowflakes lead to such
valuable scientific deductions?"

"In general equal pellets when collected in any vessel, come to a mutual agreement in two modes according to the two modes of arranging them in a plane," Kepler wrote, here substituting generic spherical pellets for the loculi. Equalsized spherical pellets squeezed into a plane will adopt either a three- or a fourcornered pattern:

Now if you proceed to pack the solid bodies as tightly as possible, and set the files that are first arranged on the level on top of others, layer on layer, the pellets will either be squared...or in triangles. If squared, either each single pellet of the upper range will rest on a single pellet of the lower, or, on the other hand, each single pellet of the upper range will settle between every four of the lower. In the former mode any pellet is touched by four neighbors in the same plane, and by one above and one below, and so on throughout, each touched by six others. The arrangement will be cubic, and the pellets, when subjected to pressure, will become cubes. But this will not be the tightest pack. In the second mode not only is every pellet touched by its four neighbors in the same plane, but also by four in the plane above and four below, and so throughout one will be touched by twelve, and under pressure spherical pellets will become rhomboid....The packing will be the tightest possible, so that in no other arrangement could more pellets be stuffed into the same container. (J. Kepler, The Six-Cornered Snowflake, trans. by C. Hardie [Oxford at the Clarendon Press, London, 1966] p. 15)

Having thus analyzed the square arrangement, Kepler went on to analyze the triangular one, noting that a spherical pellet in an upper layer rests either directly on another pellet underneath, or in the hollow formed by three underlying pellets. The latter case, he showed, is equivalent to the closest-packing arrangement discussed in the preceding paragraph. If a pyramid of pellets is built in this triangular arrangement, and then the pyramid is turned on its apex so that a flat side is facing up, removal of one pellet from this face will reveal four pellets underneath. Again, any pellet will have twelve nearest neighbors.

"Thus in the closest pack in three dimensions, the triangular pattern cannot exist without the square, and vice versa," Kepler wrote. "It is therefore obvious that the loculi of the pomegranate are squeezed into the shape of a solid rhomboid; the demands of their matter coincide with the proportions of their growth."

Thus it was by analyzing the pomegranate, and not the snowflake of the title, that Kepler succeeded in describing the basic concepts of crystallography. Of equal importance scientifically, he ascribed the arrangement of the chambers in the fruit to "the demands of their matter," not to any life force or universal spirit.

He was not able to do so with the snowflake. In the end, after examining the issue from many angles, Kepler surrendered the shape of the snowflake to the "formative faculty" of the Earth, and assigned further work on the problem to the chemists.

Having served its purpose as a New Year's gift to Johannes Matthäus Wacker von Wackerfels, the essay was published in Frankfurt in 1611 in its original Latin. Only after the late Cyril Stanley Smith of the Massachusetts Institute of Technology recommended the largely forgotten text to Whyte was it translated into English by Colin Hardie of Magdalen College, Oxford, and published again in 1966. The publication of the English version revealed to a mass audience that Johannes Kepler, known mostly for his three laws of planetary motion, was also a pioneer in the field of materials science.

TIM PALUCKA

FOR FURTHER READING: Johannes Kepler [De Nive Sexangula (Godfrey Tampach, Frankfort on Main, 1611] The Six-Cornered Snowflake, trans. by C. Hardie with a foreword by L.L. Whyte (Oxford at the Clarendon Press, London, 1966); C. Schneer, "Kepler's New Year's Gift of a Snowflake," Isis 51 (1960) p. 531; and M. von Laue, commentary in International Tables for X-Ray Crystallography, Vol. I, edited by N.F.M. Henry and K. Lonsdale (International Union of Crystallography, England, 1952). 